\title{
P-119
}

\section{Application of Molecular Imprinted Polymer Solid Phase Extraction (MISPE) in the Extraction of Caffeine From Coffee}

\author{
Rozita Osman*, Norashikin Saim, Nurdiyana Mohd Anuar and Siti Norbayu Mohd Subari \\ Faculty of Applied Sciences, Universiti Teknologi MARA, 40450 Shah Alam, Malaysia, \\ E-mail: rozit471@salam.uitm.edu.my
}

Caffeine is naturally found in plant products such as coffee beans, cocoa beans and tea leaves. In this study, caffeine MIP was synthesized by dissolving $1 \mathrm{mmol}$ caffeine and $4 \mathrm{mmol}$ monomer, methacrylic acid (MAA) and 1mmol ethylene glycol dimethacrylate (EDMA) as the crosslinker and benzoyl peroxide (BOP) as the initiator. Molecular imprinted polymers (MIP) exhibits significantly high affinity and selectivity for the target analytes used as the template than for similar molecules, including closely related isomers. The efficiency of extraction using caffeine MIP as sorbents in solid phase extraction (SPE) was evaluated. Good recovery $(91.6 \%)$ and low percent RSD $(0.9 \%)$ was obtained. The MISPE technique was applied in the extraction of caffeine from various coffee samples. Based on the excellent selectivity, MIP is a promising approach for the extraction of compounds from a complex matrix such as herbal extract.

Keywords: Molecular Imprinted Polymers, Solid Phase Extraction, Caffeine, Coffee. 